\title{
Early gastric cancer combined with multiple metachronous osteosclerotic bone and bone marrow metastases that responded to chemoradiotherapy
}

\author{
Masaaki Saito • Hirokazu Kiyozaki • \\ Fumihiro Chiba $\cdot$ Osamu Takata $\cdot$ Takayoshi Yoshida $\cdot$ \\ Chio Shuto $\cdot$ Shigeki Yamada $\cdot$ Fumio Konishi
}

Received: 22 September 2010/Accepted: 17 May 2011/Published online: 14 June 2011

(C) The International Gastric Cancer Association and The Japanese Gastric Cancer Association 2011

\begin{abstract}
We report a 75-year-old woman who suffered multiple metachronous osteosclerotic bone metastases 4 years after a distal gastrectomy for early gastric cancer (EGC). The primary tumor was a poorly differentiated adenocarcinoma, which had invaded the submucosal layer, and only one lymph node metastasis was noted. To the best of our knowledge, cases of EGC combined with metachronous osteosclerotic multiple bone and bone marrow metastases that respond to chemoradiotherapy are very rare. In this case, the multiple bone metastases were diagnosed 4 years after surgery. The patient's metastatic tumor was successfully treated using S-1, paclitaxel, and camptothecin, with subsequent irradiation. The patient survived for 24 months after the treatment, without having any major symptoms.
\end{abstract}

Keywords Stomach $\cdot$ Cancer $\cdot$ Submucosal $\cdot$ Multiple bone metastases $\cdot$ Chemoradiotherapy

M. Saito $(\bowtie) \cdot$ H. Kiyozaki · F. Chiba · O. Takata ·

T. Yoshida $\cdot$ F. Konishi

Department of Surgery, Jichi Medical University Saitama

Medical Center, Amanuma-cho 1-847,

Omiya-ku, Saitama, Japan

e-mail: msaito@jichi.ac.jp

S. Yamada

Department of Pathology, Jichi Medical University Saitama

Medical Center, Saitama, Japan

C. Shuto

Department of Surgery, Ogano Municipal Central Hospital,

Saitama, Japan

\section{Introduction}

Recently, the clinical outcome of early gastric cancer (EGC) has gradually been improving, and the 5-year survival rate for EGC patients has been reported to exceed $85 \%$ in most studies [1-3]. However, some cases of EGC are associated with distant metastasis $[4,5]$.

The most common sites of gastric cancer recurrence are the peritoneum, local lymph nodes, and liver. Bone metastases are very rare and are only observed in $0.99-2.1 \%$ of gastric cancer cases $[6,7]$. The occurrence of multiple metachronous bone metastases from EGC after a 4-year disease-free interval following gastrectomy is even rarer.

Gastric cancer bone metastasis often involves multiple lesions and is usually associated with metastasis to other organs; therefore, the main treatment option for gastric cancer is chemotherapy.

We report a 75-year-old woman who suffered with multiple metachronous bone metastases 4 years after undergoing a distal gastrectomy for EGC.

Our case can be considered to be a rare case because the patient had osteosclerotic bone and bone marrow involvement without the disease spreading to other organs.

\section{Case report}

A 75-year-old woman presented to a local hospital complaining of epigastric pain and was diagnosed with suspected gastric cancer. As a result, she was referred to our department for surgical treatment. She had no relevant personal or family history. Endoscopy revealed a depressed lesion with central ulceration in the lower body of the stomach. Biopsies were taken from this area, and pathology 
demonstrated poorly differentiated gastric adenocarcinoma and signet ring cells (Fig. 1). Her chest and abdominal $\mathrm{X}$-ray findings were normal, and an ultrasound examination and computed tomography (CT) scans of the abdomen revealed no metastases to the liver, and no involvement of adjacent organs.

The patient was diagnosed with EGC and underwent distal gastrectomy with limited lymph node dissection $(\mathrm{D} 1+\beta)$ on May 30,2002 . The primary tumor was located in the lesser curvature of the lower body of the stomach and had only invaded the submucosa (SM1). Histology showed the presence of a diffuse-type gastric adenocarcinoma confined to the submucosa (SM1) and signet ring cells (Fig. 2). Only one (perigastric lymph node) of 33 dissected lymph nodes demonstrated metastatic adenocarcinoma disease, involving signet ring cells, and no venous invasion was seen.

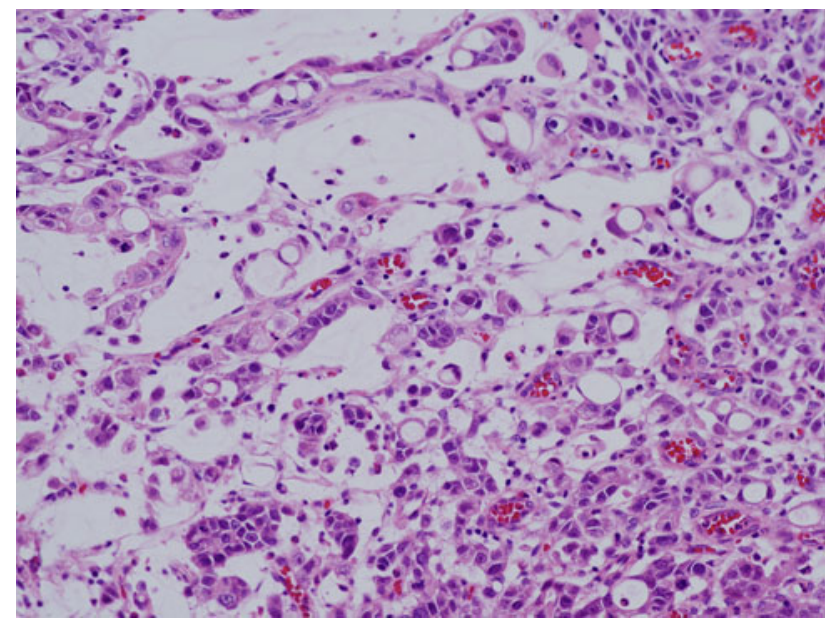

Fig. 1 Pathology demonstrates poorly differentiated gastric adenocarcinoma and signet ring cells $(\mathrm{H} \& \mathrm{E} \times 30)$

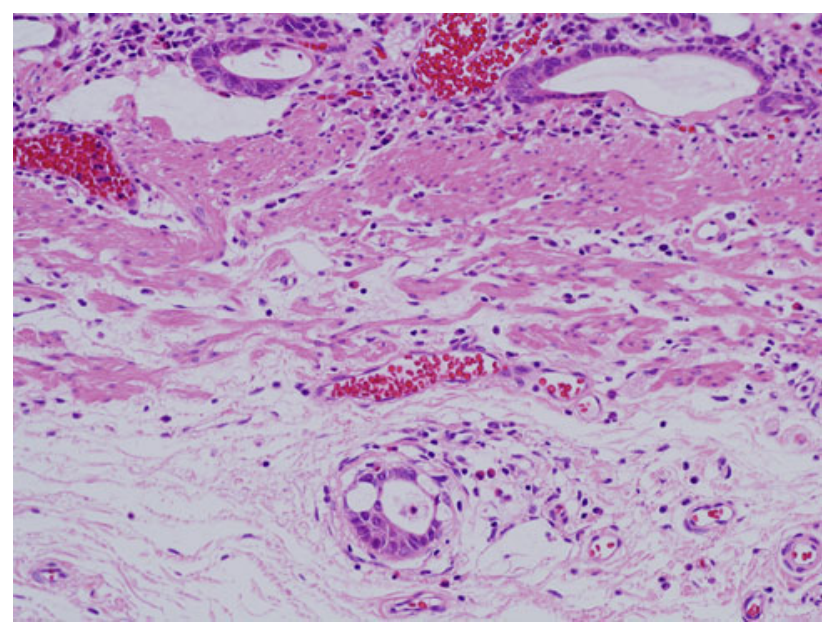

Fig. 2 Histology showed the presence of a diffuse-type gastric adenocarcinoma confined to the submucosa (SM1) and signet ring cells $(H \& E \times 30)$
The patient's postoperative course was uneventful, and she was discharged from our hospital 11 days after the surgery. Following the surgery, no adjuvant chemotherapy was administered.

Postoperative annual surveillance revealed no evidence of recurrence for the next 3 years. However, approximately 4 years after the surgery, her carcinoembryonic antigen (CEA) and carbohydrate antigen (CA) 19-9 levels rose to $1314 \mathrm{ng} / \mathrm{mL}$ and $160,600 \mathrm{U} / \mathrm{mL}$, respectively.

Because no recurrent lesion was detected on whole-body CT, fluorodeoxyglucose positron emission tomography (FDG-PET) was performed, and abnormal diffuse accumulation was observed only in the lumbar vertebrae. A whole-body bone (99m Tc MDP-99m Tc-methylene diphosphonate) scan showed increased diffuse uptake throughout the body (a so-called 'beautiful bone scan') (Fig. 3), while magnetic resonance imaging (MRI) of the thoracic and lumbar spine confirmed the presence of osteosclerotic metastatic lesions in the areas that showed increased uptake on the bone scan (Fig. 4).

The results of these imaging examinations were suggestive of multiple bone metastases. In order to exclude the presence of another primary lesion such as one more commonly associated with this type of metastasis (i.e., breast or uterine cancer), the patient underwent a clinical breast examination and ultrasonography. Both examinations were negative. Therefore, the presence of another primary lesion was excluded.

A bone biopsy was undertaken from the superior iliac crest. Histology showed the presence of a diffuse-type adenocarcinoma with myxomatous stroma in the bone and bone marrow tissue, which resembled the histology of the previous gastric cancer (Fig. 5). Therefore, the patient was diagnosed with multiple metachronous bone and bone marrow metastases from EGC.

Treatment with S-1 was started in February 2006 at the local hospital, according to the following regimen: daily oral administration of $100 \mathrm{mg} /$ body/day S-1 for 28 days. Four months after the initiation of therapy, her CEA and CA19-9 levels had decreased to within the normal ranges. No severe adverse effects associated with this therapy were observed.

However, 6 months after the initiation of therapy, her CEA and CA19-9 levels started to increase again. Therefore, she was given second-line chemotherapy in August 2006, with the following regimen: paclitaxel was intravenously administered $\left(80 \mathrm{mg} / \mathrm{m}^{2}\right)$ on days 1,8 , and 15 . The patient received two courses of this chemotherapy, but no chemotherapeutic effect on her tumor marker levels was observed. Therefore, the patient received third-line chemotherapy, in December 2006, with the following regimen: intravenous administration of irinotecan (CPT-11) $\left(100 \mathrm{mg} / \mathrm{m}^{2}\right)$ on days 1,8 , and 15 . The patient received eight courses of this chemotherapy, but again her CEA and CA19-9 levels did not decrease. The patient developed 
Fig. 3 Whole-body bone $(99 \mathrm{~m}$ Tc MDP) scan showed increased diffuse uptake throughout the body
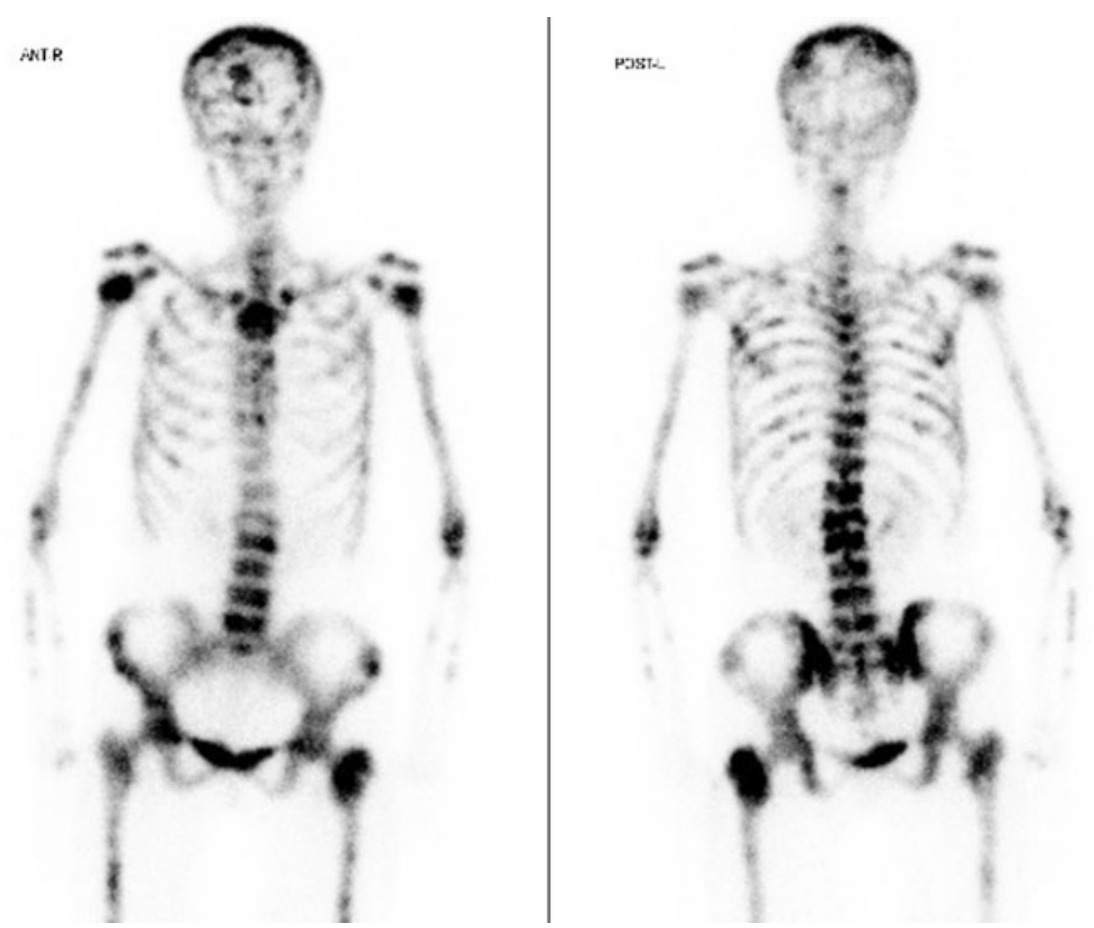

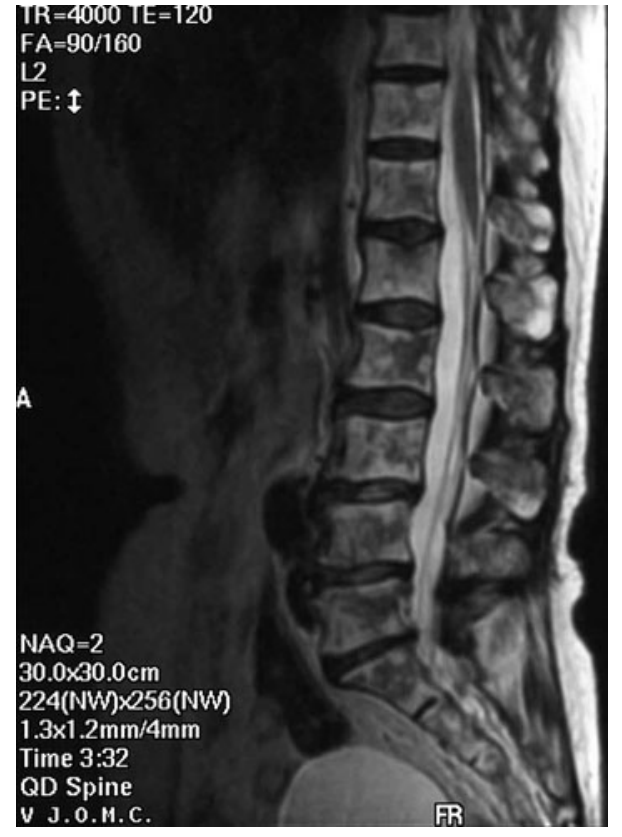

Fig. 4 Magnetic resonance imaging (MRI) of the thoracic and lumbar spine confirmed the presence of osteosclerotic metastatic lesions in the areas that demonstrated increased uptake on the bone scan

lower back pain and was then referred back to our hospital for further treatment.

Extracorporeal radiotherapy (2000 cGy) was given to the right ileum (palliative radiotherapy for symptomatic metastatic bone metastases). After treatment with radiotherapy and analgesics, the patient's symptoms were

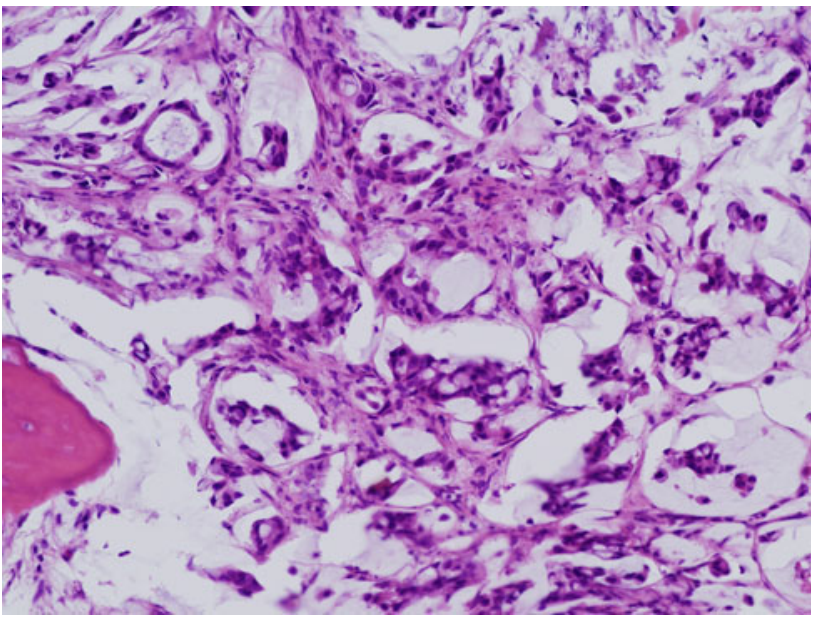

Fig. 5 Pathology demonstrates the presence of diffuse-type adenocarcinoma with a myxomatous stroma in the bone and bone marrow tissue, which resembled the histology of the previous gastric cancer $(\mathrm{H} \& \mathrm{E} \times 30)$

relieved. The patient was referred to a local hospital, and she died of aspiration pneumonia 78 months after the initial gastric surgery (30 months after the initiation of chemotherapy). No autopsy was performed.

\section{Discussion}

According to the WHO cancer statistics, the incidence and mortality rate of gastric cancer are the fourth and second highest in the world, respectively $[8,9]$. 
In the Gastric cancer treatment guidelines produced by the Japanese Gastric Cancer Association, EGC is defined as gastric carcinoma confined to the mucosa and submucosa regardless of the presence or absence of lymph node metastasis [10]. The 5-year-survival rate for EGC has been reported to be as high as $85 \%$ [1-3]. This is probably due to the low rate of metastasis to extragastric sites, making the early detection of gastric cancer important for improving its prognosis.

It has been reported that the most common form of gastric cancer recurrence, including that of advanced cancer, is peritoneal dissemination, followed by local recurrence, hepatic metastasis, and distant metastasis, with bone metastasis being rare. Seto et al. [11] reported that the most common site of bone metastasis was the spine and that diffuse bone metastasis (such as that found in the present patient) was detected in approximately $20 \%$ of patients. The prognosis of bone metastasis is generally poor, and some patients develop disseminated bone marrow carcinosis combined with disseminated intravascular coagulation (DIC) and die early [12]. Ohyama et al. [13] reported that the 3- and 6-month survival rates after the diagnosis of bone metastasis were low, at 33 and $11 \%$, respectively.

Characteristically, gastric cancer metastasizing to the bone tends to develop at a young age, to involve histologically undifferentiated adenocarcinoma (particularly signet-ring cell carcinoma), to be highly angioinvasive, and to frequently involve advanced-stage disease combined with lymph node metastasis [5]. It has been reported that, among gastric cancers, lymph node or distant metastasis is common in poorly differentiated adenocarcinoma [14].

Our patient's disease was histologically undifferentiated and involved lymph node metastasis, but was in its early stages, and had developed at an advanced age. These characteristics differ from those described above.

Generally, patients with EGC combined with bone metastasis almost always undergo a detailed examination after the onset of symptoms, leading to a diagnosis. Reportedly, dorsolumbar pain is the most frequent symptom of bone metastasis, but asymptomatic cases such as the present case are not rare. In this case, the patient's CEA and CA19-9 levels rose, and abnormal accumulation was noted on FDG-PET and bone scintigraphy. As a result, multiple bone metastases were diagnosed. FDG-PET and bone scintigraphy are useful for the regional diagnosis of recurrent lesions, especially for bone metastases.

Our patient had no symptoms of the bone metastases, but her platelet count and serum alkaline phosphatase (ALP) and lactic dehydrogenase (LDH) levels were elevated, leading to a diagnosis of multiple bone metastases.

There are two patterns of bone metastasis, the osteolytic type and the osteosclerotic type. Osteosclerotic bone metastases are frequent in breast and prostate cancer. In EGC, bone metastases are very rare, and osteosclerotic metastases are even rarer, and this is only the seventh case of osteosclerotic bone metastases from gastric cancer reported in the English-language literature. Our case can be considered to be a very rare case because the patient had osteosclerotic bone metastases without the disease spreading to other organs.

Bone metastasis of gastric cancer often involves multiple metastases and is usually associated with metastasis to other organs; therefore, the main treatment option is chemotherapy [15]. However, patients with a solitary metastasis or symptoms such as pain commonly receive radio- and chemotherapy. Our patient underwent radiotherapy for painful lumbar spinal metastasis and achieved pain relief. The usefulness of strontium chloride (89Sr) for reducing the pain caused by bone-forming bone metastases from prostate cancer has been reported [16]. Strontium chloride accumulates and reduces pain in regions with enhanced bone-forming activity. Therefore, it is appropriate for pain reduction in cases involving multiple bone metastases because it accumulates in bone metastatic lesions throughout the body following a single intravenous injection. Strontium chloride was not used to treat the present patient because her cancer pain was successfully controlled through the use of oral analgesics and by irradiating the painful region. However, this agent may be favorably indicated for multiple bone-forming bone metastases from solid cancer, similar to those in the present case.

Recently, chemotherapy with S-1 for recurrent gastric cancer was found to produce a favorable outcome. According to the SPIRITS study, the standard first-choice treatment for recurrent gastric cancer in Japan is S-1/cisplatin (CDDP) therapy [17].

Due to her advanced age and coexisting disease, the present patient did not receive $\mathrm{S}-1$ plus CDDP, but rather was treated with $\mathrm{S}-1$ alone, which effectively controlled her bone metastases. During this chemotherapy, no hematological or gastrointestinal toxicity was observed; therefore, the therapy was successfully continued and was effective at maintaining the patient's quality of life (QOL).

Because even EGC may metastasize to distant sites, the optimal treatment plan requires systematic investigation. It is suggested that the survival of gastric cancer patients with severe bone metastasis can be improved by continuous chemotherapy.

In patients with histologically poorly differentiated gastric cancer combined with lymph node metastasis, strict follow-up after surgery is necessary, even at an early stage, as was performed in the present patient. When a recurrent lesion cannot be confirmed by conventional CT, but back pain and elevated tumor marker levels are present, bone 
metastasis should be suspected, and a whole-body bone scan should be performed as part of the follow-up examination.

\section{References}

1. Nakajima T. Gastric cancer treatment guidelines in Japan. Gastric Cancer. 2002;5(1):1-5.

2. Santoro E, Garofalo A, Scutari FA, Carlini M, Zanarini T. Multicenter retrospective study of 3,024 patients operated on for stomach cancer in Italy. Epidemiology, surgical treatment and survival. Ann Gastroenterol Hepatol (Paris). 1991;27(4):167-71.

3. Oliveira FJ, Ferrao H, Furtado E, Batista H, Conceicao L. Early gastric cancer: report of 58 cases. Gastric Cancer. 1998;1(1):51-6.

4. Ichiyoshi Y, Toda T, Minamisono Y, Nagasaki S, Yakeishi Y, Sugimachi K. Recurrence in early gastric cancer. Surgery. 1990;107(5):489-95.

5. Sano T, Sasako M, Kinoshita T, Maruyama K. Recurrence of early gastric cancer. Follow-up of 1475 patients and review of the Japanese literature. Cancer. 1993;72(11):3174-8.

6. Guadagni S, Catarci M, Kinoshita T, Valenti M, De Bernardinis G, Carboni M. Causes of death and recurrence after surgery for early gastric cancer. World J Surg. 1997;21(4):434-9.

7. Yoshikawa K, Kitaoka H. Bone metastasis of gastric cancer. Jpn J Surg. 1983;13(3):173-6.

8. Yako-Suketomo H, Katanoda K. Comparison of time trends in stomach cancer mortality (1990-2006) in the world, from the WHO mortality database. Jpn J Clin Oncol. 2009;39(9):622-3.

9. Imamura Y, Yoshimi I. Comparison of cancer mortality (stomach cancer) in five countries: France, Italy, Japan, UK and USA from the WHO Mortality Database (1960-2000). Jpn J Clin Oncol. 2005;35(2):103-5.

10. Shimada Y. JGCA (The Japanese Gastric Cancer Association). Gastric cancer treatment guidelines. Jpn J Clin Oncol. 2004;34(1):58.

11. Seto M, Tonami N, Koizumi K, Sui O, Hisada K. Bone metastasis in gastric cancer-clinical evaluation of bone scintigrams. Kaku Igaku. 1983;20(6):795-801.

12. Hironaka SI, Boku N, Ohtsu A, Nagashima F, Sano Y, Muto M, Fujii T, Tajiri H, Yoshida S. Sequential methotrexate and 5-fluorouracil therapy for gastric cancer patients with bone metastasis. Gastric Cancer. 2000;3(1):19-23.

13. Ohyama S, Yonemura Y, Taniya T, Koyasaki N, Hashimoto T, Sawa T, Shima Y, Miwa K, Miyazaki T. Bone metastasis of gastric cancer. Jpn J Gastroenterol Surg. 1987;20(11):2516-20.

14. Kodama Y, Inokuchi K, Soejima K, Matsusaka T, Okamura T. Growth patterns and prognosis in early gastric carcinoma. Superficially spreading and penetrating growth types. Cancer. 1983;51(2):320-6.

15. Anagnostopoulos G, Sakorafas GH, Kostopoulos P, Margantinis G, Tsiakos S, Pavlakis G. Early (mucosal) gastric cancer with synchronous osteosclerotic bone metastases: a case report. Eur J Cancer Care (Engl). 2010;19(4):554-7.

16. Kraeber-Bodere F, Campion L, Rousseau C, Bourdin S, Chatal JF, Resche I. Treatment of bone metastases of prostate cancer with strontium-89 chloride: efficacy in relation to the degree of bone involvement. Eur J Nucl Med. 2000;27(10):1487-93.

17. Koizumi W, Narahara H, Hara T, Takagane A, Akiya T, Takagi M, Miyashita K, Nishizaki T, Kobayashi O, Takiyama W, Toh Y, Nagaie T, Takagi S, Yamamura Y, Yanaoka K, Orita H, Takeuchi M. S-1 plus cisplatin versus S-1 alone for first-line treatment of advanced gastric cancer (SPIRITS trial): a phase III trial. Lancet Oncol. 2008;9(3):215-21. 